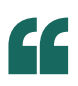

\section{The critical}

challenge was

to preserve

the isoporous

morphology of

these hybrid

films

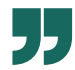

$\Rightarrow$ GAS SENSORS

\title{
Pores for effect
}

When it comes to gas sensing, isoporous films are useful because their large surface area gives them plenty of opportunity to interact with small molecules. However, these films are typically formed from organic polymers with low electrical conductivity, making the films unsuitable for incorporation into devices. Writing in Angewandte Chemie International Edition, Klaus-Viktor Peinemann, Tom Wu and co-workers describe a new material that combines the porosity of polymer films with the conductivity of carbon nanotubes (CNTs). Their judiciously chosen diblock copolymer polystyrene- $b$-poly(4-vinyl pyridine) (PS- $b$-P4VP) served as a perfect host for CNTs, producing a humidity sensor with a response time of less than 1 second.

The team had earlier developed a method for manufacturing isoporous films based on block copolymer selfassembly, an approach they extended and optimized to incorporate CNTs. "The critical challenge was to preserve the isoporous morphology of these hybrid films," says team member Rahul Shevate. The process is simple: CNTs are first dispersed in $\mathrm{DMF} / \mathrm{THF} / 1,4$-dioxane, after which PS- $b$-P4VP is dissolved in the mixture; this solution is then partially evaporated on a glass surface before being immersed in water, leading to precipitation of the composite. Scanning electron microscope and atomic force microscope images of the resulting films show ordered patterns of $20 \mathrm{~nm}$ pores - an ideal morphology for humidity sensing. In addition, the self-assembly process causes the pores to be lined by the hydrophilic P4VP blocks in PS- $b$-P4VP. "This should lead to fast sorption and desorption of $\mathrm{H}_{2} \mathrm{O}$ when exposed to changes in humidity," says Peinemann. Transmission electron

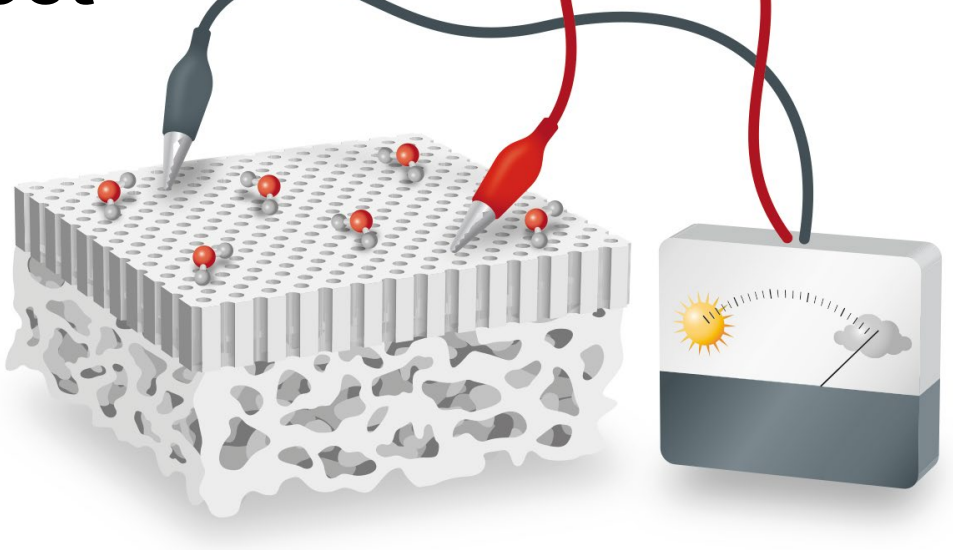

micrographs of the films also show that the CNTs are dispersed, which the team attributes to stabilizing interactions between P4VP and the CNT sidewalls, both in the form of $\pi-\pi$ interactions and interactions involving $\mathrm{N}$ valence lone pairs. Raman spectra of the composite feature a radial breathing band and a G band, consistent with the CNTs retaining their electronic properties when embedded in the film.

Proof that the sensors work comes from the measured electrical conductivity, which increases exponentially with increasing relative humidity. At a relative humidity of $95 \%$, the response time is only 0.3 seconds. By comparison, PS- $b$-P4VP films without CNTs have lower electrical conductivity and a 12-fold slower response. The diblock copolymer is also essential, and sensors made of drop-casted CNT films show only a small change in electrical conductivity with relative humidity. The team also tested films with CNTs embedded in the random copolymer poly-2-vinylpyridineco-styrene, which is chemically similar to the diblock copolymer PS- $b$-P4VP but does not form ordered pores. The conductivity of these films depends very little on relative humidity.

The results of Peinemann and co-workers gave them clues about how their films conduct electricity. The team proposes that the movement of both protons and electrons enables three conduction mechanisms. "While the traditional Grotthuss mechanism ( $\mathrm{H}^{+}$hopping) is at play, another contribution comes from the charge transfer from the block copolymer to the CNTs. In addition, $\mathrm{e}^{-}$transfer from the vapour to the CNTs also contributes to the overall response," explains Peinemann.

The team now hopes to build sensors that can selectively quantify volatile organics with high sensitivity. In the present design, humidity interferes with the binding of volatile organics. "We plan to fabricate sensors based on isoporous films consisting of multiple fillers, each with a distinct selectivity," says Peinemann. This might be achieved by lining pores with cyclodextrins, whose hydrophobic interiors bind organics. "We envision that these fillers will selectively detect various analytes simultaneously in multi-sensing array devices," concludes Peinemann.

Zoe Budrikis, Associate Editor, Nature Reviews Physics.

ORIGINAL ARTICLE Shevate, R. et al. Embedding $1 \mathrm{D}$ conducting channels into $3 \mathrm{D}$ isoporous polymer films for high performance humidity sensing. Angew. Chem. Int. Ed. https://doi.org/ 10.1002/anie.201804656 (2018) 